\title{
Riqueza de las familias Agavaceae y Nolinaceae en México
}

\author{
ABISAÍ GARCÍA-MENDOZA ${ }^{1}$ Y RAQUEL GALVÁN V. ${ }^{2}$ \\ ${ }^{\prime}$ Jardín Botánico, IB-UNAM. Apdo. Postal 70-614, Del. Coyoacán, 04510 México, D.F. \\ ${ }^{2}$ Escuela Nacional de Ciencias Biológicas, IPN. Apdo. Postal 17-564, Del. M. Hidalgo, 11410 México, D.F.
}

\begin{abstract}
Resumen. Se muestra la distribución de las familias Agavaceae y Nolinaceae en América y México. Para México se determinó la presencia de 402 taxa, 342 de ellos pertenecen a los géneros Agave, Beschorneria, Furcraea, Hesperaloë, Manfreda, Polianthes, Prochnyanthes y Yucca de la familia Agavaceae, en tanto que 60 corresponden a los géneros Beaucarnea, Calibanus, Dasylirion y Nolina de la familia Nolinaceae. Se presenta también la lista actualizada de las especies de ambas familias, ordenadas alfabéticamente. Para cada taxon se señala su distribución por estado y por provincia florística. Los estados más ricos son: Oaxaca con 63 taxa, Durango con 52, Puebla con 50, San Luis Potosí y Sonora con 47 y Chihuahua con 45 . En cuanto a las provincias florísticas con un número mayor de taxa están: las Serranías Meridionales, Sierra Madre Occidental y Altiplanicie. Para México, hasta el momento, se han realizado cinco floras regionales y cuatro listados florísticos, en los que se aborda el estudio de las Agavaceae y Nolinaceae a diferentes niveles. Los géneros Agave, Beaucarnea, Beschorneria, Manfreda y Prochnyanthes han sido objeto de tratamientos taxonómicos; otros como Dasylirion, Furcraea y Polianthes se encuentran en diferentes etapas de desarrollo, en tanto que Calibanus, Hesperaloë, Nolina, Yucca y varios grupos de Agave, requieren una revisión actualizada. Se mencionan datos sobre la representación de los taxa a nivel de los dos principales herbarios de México (ENCB y MEXU), los cuales en conjunto suman 220 especies, avaladas por 3,593 ejemplares, con un promedio de 16.3 por taxon. Sin embargo, es necesario impulsar las colectas de herbario y de jardines botánicos, para no sólo respaldar los trabajos florísticos y taxonómicos, sino para apoyar investigaciones ecológicas, etnobotánicas, conservacionistas, etc. Estas acciones nos permitirán tener un conocimiento más profundo de la biología de las Agavaceae y Nolinaceae.

Palabras clave: Agavaceae, fitogeografía, endemismo, México, Nolinaceae.
\end{abstract}

Abstract. We show the distribution of the Agavaceae and Nolinaceae families in the Americas and in Mexico. For Mexico we determined the existence of 402 taxa of which 342 belong to the genera Agave, Beschorneria, Furcraea, Hesperaloë, Manfreda, Polianthes, Prochnyanthes and Yucca in the Agavaceae family, while 60 are from the genera Beaucarnea, Calibanus, Dasylirion and Nolina from the Nolinaceae family. We also show an updated list of the species from both families arranged alphabetically. For each taxon we describe their distribution by state and by floristic provinces. The richest states are Oaxaca with 63 taxa, Durango with 52, Puebla with 50, San Luis Potosí and Sonora with 47 and Chihuahua with 45 . The floristic provinces with higher number of taxa are: The Meridional Sierras, the Sierra Madre Occidental and the Central Mexico Highlands. At the moment, for Mexico, five regional floras have been finished that study the Agavaceae and Nolinaceae at different levels. The genera Agave, Beaucarnea, Beschorneria, Manfreda and Prochnyanthes have had recent taxonomic treatments; others like Dasylirion, Furcraea and Polianthes are in different stages of development, while Calibanus, Hesperaloë, Nolina, Yucca and several groups of Agave require an update. We review data on the representation of the taxa in two of the main herbaria in Mexico (ENCB and MEXU), which comprise 220 species, backed by 3,593 vouchers, with an average of 16.3 vouchers per taxon. Neverthless it is necessary to increase the collection for herbarium and botanical gardens, to not only to back up flc ristic and taxonomic research, but also to support research in ecology, ethnobotany, conservation biology, etc. These actions will allow us to have a better knowledge of the biology of the Agavaceae and Nolinaceae.

Key words: Agavaceae, phytogeography, endemics, Mexico, Nolinaceae.

\section{ANTECEDENTES}

La familia Agavaceae fue propuesta por Endlicher (1836$1840,1841)$, quien tomó como tipo nomenclatural al género Agave, incluyendo además al género Furcraea. Salisbury (1866) divide la familia en dos secciones, la de ovario súpero con el género Yucca y la sección de ovario ínfero con los géneros Agave, Furcraea, Littaea, Manfreda y Polianthes. Lotsy (1911) mantiene la familia sólo con los géneros de ovario ínfero, aunque adiciona Beschorneria, Bravoa y Doryanthes.
Bentham y Hooker (1883), Pax (1887), Baker (1888) y Pax y Hoffman (1930) tratan a los géneros de la familia en forma tradicional, es decir aquellos de ovario ínfero dentro de la familia Amaryllidaceae y los de ovario súpero en la familia Liliaceae. Hutchinson $(1934,1959)$ crea el orden Agavales y amplía la circunscripción de las Agavaceae a 19 géneros, incluidos en seis tribus. A partir de esta fecha se ha levantado una serie de controversias tanto sobre su validez como acerca de su delimitación genérica.

Investigaciones en citología, contemporáneas a Hutchinson y realizadas por McKelvey y Sax (1933), Whitaker 
(1934), Satô (1935) y Granick (1944) describen un cariotipo bimodal de $\mathrm{n}=30$ (cinco cromosomas grandes y 25 pequeños), común a los géneros cercanos a Yucca y Agave. Mientras que Nolina, Calibanus y Dasylirion tienen sus cromosomas más o menos del mismo tamaño y su cariotipo es de $\mathrm{n}=19$ (Cave, 1964); Dracaena y Sansevieria de $\mathrm{n}=$ 20 (Granick, 1944); Phormium de $\mathrm{n}=16$ (Darlington y Wylie, 1955, en Gómez Pompa, 1963) y Doryantes de $\mathrm{n}=$ 24 (Cave, 1964). Sólo el género Hosta de China y Japón tiene el cariotipo bimodal de Agavaceae (Takhtajan, 1980) aunque sus relaciones filogenéticas parecen estar más cercanas al género Clorophytum de distribución africana (Eguiarte et al., 1994).

Posterior a 1960 se propusieron varios rearreglos taxonómicos en cuanto a las familias Liliaceae, Amaryllidaceae y Agavaceae. Satô (1942), Wunderlich (1950) y Takhtajan (1980) plantean la formación de un mayor número de familias o grupos. Gómez-Pompa (1963), Gómez-Pompa et al. (1971), Thorne (1968) y Mc Vaugh (1989) proponen la unificación de Liliaceae y Amaryllidaceae dentro de la primer familia. Cronquist $(1968,1981)$ propone la unión de las dos familias anteriores, pero con Agavaceae aparte. Lawrence (1951), Porter (1967) y Benson (1979) sugieren tratar Amaryllidaceae y Liliaceae por separado, sin incluir Agavaceae.
En un sistema de clasificación más reciente Dahlgren et al. (1985), tomando en cuenta numerosos caracteres (cariotípicos, anatómicos, químicos, biogeográficos, etc.) y con base en un análisis cladístico, presentan un arreglo estricto de las Agavaceae, en el cual reconocen dos subfamilias: Yuccoideae con ovario súpero que incluye a los géneros Yucca y Hesperaloë y Agavoideae con los géneros Agave, Beschorneria, Furcraea, Manfreda, Polianthes y Prochnyanthes, mientras que la familia Nolinaceae contiene a los géneros Beaucarnea, Calibanus, Dasylirion y Nolina. Esta clasificación ha sido apoyada con estudios recientes sobre ADN del cloroplasto, realizados por Eguiarte et al. (1994) y Bogler y Simpson (1995).

Las diferencias morfológicas gruesas para diferenciar ambas familias es que las Agavaceae tienen semillas con fitomelano que les da la pigmentación negra, flores grandes, bisexuales y frutos triloculares con más de dos óvulos, mientras que las Nolinaceae tienen semillas sin fitomelano, inflorescencias poligamodioicas, flores pequeñas, unisexuales y lóculos del fruto con 1-2 (-6) óvulos.

\section{DISTRIBUCIÓN}

\section{Agavaceae}

La familia Agavaceae es endémica de América; se distribuye desde el sur de Canadá, México, Centroamérica, norte de Sudamérica (siguiendo principalmente la cadena montañosa

Cuadro 1. Número de especies y taxa infraespecíficos de Agavaceae y Nolinaceae en México y el mundo.

AGAVACEAE

\begin{tabular}{|c|c|c|c|c|c|c|}
\hline GENEROS & $\begin{array}{l}\text { NO. TOTAL DE } \\
\text { SPP. }\end{array}$ & $\begin{array}{l}\text { NO. TOTAL } \\
\text { DE TAXA }\end{array}$ & $\begin{array}{l}\text { SPP. EN } \\
\text { MEXICO }\end{array}$ & $\begin{array}{l}\text { TAXA EN } \\
\text { MEXICO }\end{array}$ & $\begin{array}{c}\text { \% DE SPP. EN } \\
\text { MEXICO }\end{array}$ & $\begin{array}{l}\% \text { DE TAXA } \\
\text { EN MEXICO }\end{array}$ \\
\hline Agave & 166 & 207 & 125 & 161 & 75 & 78 \\
\hline Beschorneria & 7 & 8 & 7 & 8 & 100 & 100 \\
\hline Furcraea & 20 & 22 & 11 & 11 & 55 & 50 \\
\hline Hesperaloë & 3 & 3 & 3 & 3 & 100 & 100 \\
\hline Manfreda & 28 & 28 & 27 & 27 & 96 & 96 \\
\hline Polianthes & 13 & 16 & 13 & 16 & 100 & 100 \\
\hline Prochnyantes & 1 & 1 & 1 & 1 & 100 & 100 \\
\hline Yucca & 50 & 57 & 30 & 31 & 60 & 54 \\
\hline TOTAL & 288 & 342 & 217 & 258 & 75 & 75 \\
\hline
\end{tabular}

\section{NOLINACEAE}

\begin{tabular}{lccccc}
\hline GENEROS & $\begin{array}{c}\text { NO. TOTAL DE } \\
\text { SPP. }\end{array}$ & $\begin{array}{c}\text { NO. TOTAL } \\
\text { DE TAXA }\end{array}$ & $\begin{array}{c}\text { SPP. EN } \\
\text { MEXICO }\end{array}$ & $\begin{array}{c}\text { TAXA EN } \\
\text { MEXICO }\end{array}$ & $\begin{array}{c}\% \text { DE SPP. EN } \\
\text { MEXICO }\end{array}$ \\
\hline Beaucarnea & 10 & 11 & 9 & 10 & 90 \\
Calibanus & 1 & 1 & 1 & 1 & 100 \\
Dasylirion & 19 & 19 & 19 & 19 & 100 \\
Nolina & 25 & 29 & 20 & 21 & 80 \\
TOTAL & 55 & 60 & 49 & 51 & 89 \\
\end{tabular}




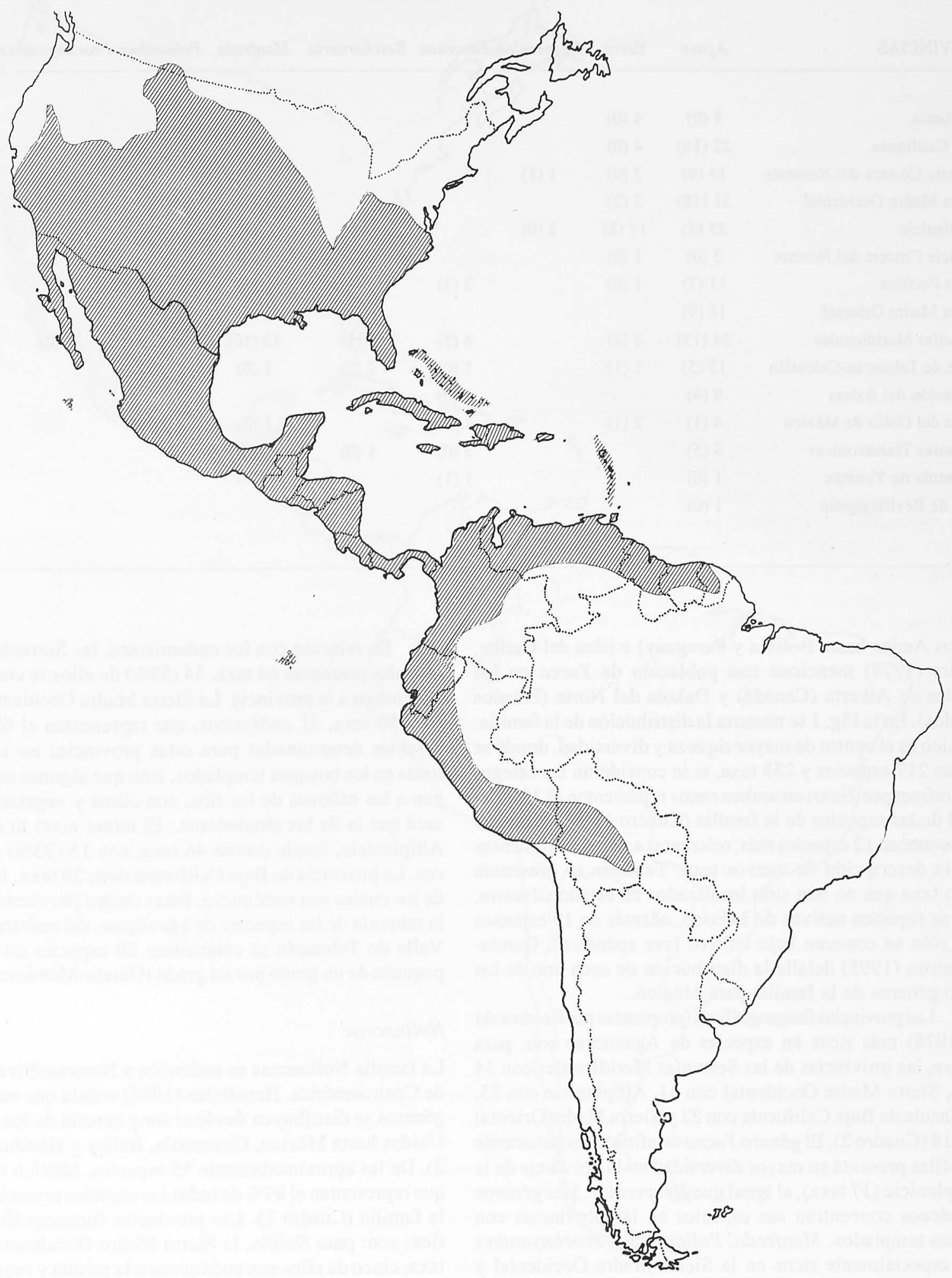


CuAdro 2. Distribución de los taxa de Agavaceae por provincia florística, entre paréntesis las endémicas a la provincia.

\section{PROVINCIAS}

Agave Yucca Hesperaloë Furcraea Beschorneria Manfreda Polianthes Prochnyanthes TOTAL

$\begin{array}{lccc}\text { California } & 5(0) & 4(0) & \\ \text { Baja California } & 22(16) & 4(0) & \\ \text { Planicie Costera del Noroeste } & 13(9) & 2(0) & 1(1) \\ \text { Sierra Madre Occidental } & 31(18) & 2(2) & \\ \text { Altiplanicie } & 23(5) & 17(8) & 2(0) \\ \text { Planicie Costera del Noreste } & 2(0) & 1(0) & \\ \text { Costa Pacífica } & 11(7) & 1(0) \\ \text { Sierra Madre Oriental } & 18(9) & \\ \text { Serranías Meridionales } & 34(13) & 2(2) \\ \text { Valle de Tehuacán-Cuicatlán } & 15(5) & 1(1) \\ \text { Depresión del Balsas } & 9(4) & \\ \text { Costa del Golfo de México } & 4(1) & 2(1) \\ \text { Serranías Transístmicas } & 9(5) & \\ \text { Península de Yucatán } & 1(0) & \\ \text { Islas de Revillagigedo } & 1(0) & \end{array}$

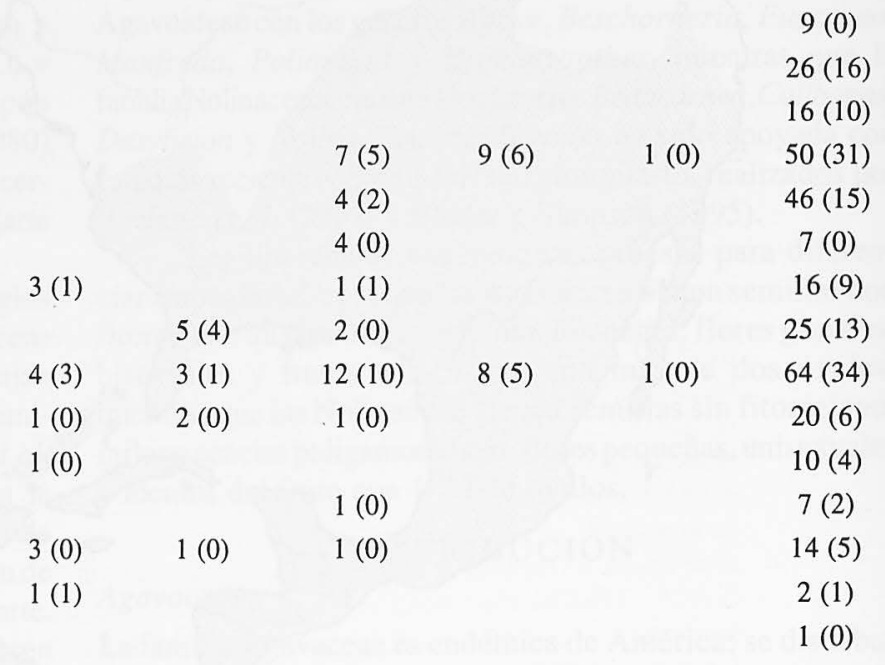

de los Andes hasta Bolivia y Paraguay) e islas del Caribe. Speirs (1979) menciona una población de Yucca en los límites de Alberta (Canadá) y Dakota del Norte (Estados Unidos). En la Fig. 1 se muestra la distribución de la familia. México es el centro de mayor riqueza y diversidad, donde se hallan 217 especies y 258 taxa, si se consideran las categorías infraespecíficas; en ambos casos representan el 75\% del total de las especies de la familia (Cuadro 1). Actualmente se reconocen 12 especies más, referentes a redescubrimientos o a la descripción de nuevos taxa. También se presentan ocho taxa que no han sido localizados en estado silvestre, que se suponen nativos de México, además de 19 especies que sólo se conocen bajo cultivo (ver apéndice). GarcíaMendoza (1995) detalla la distribución de cada uno de los ocho géneros de la familia para México.

Las provincias fitogeográficas (propuestas porRzedowski en 1978) más ricas en especies de Agavaceae son: para Agave, las provincias de las Serranías Meridionales con 34 taxa, Sierra Madre Occidental con 31, Altiplanicie con 23, península de Baja California con 22 y Sierra Madre Oriental con 18 (Cuadro 2). El género Yucca de afinidades netamente xerófilas presenta su mayor diversidad en la provincia de la Altiplanicie (17 taxa), al igual que Hesperaloë. Los géneros herbáceos concentran sus especies en las provincias con climas templados. Manfreda, Polianthes y Prochnyanthes son especialmente ricos en la Sierra Madre Occidental y Serranías Meridionales, mientras que Beschorneria lo es en la Sierra Madre Oriental. Las afinidades de Furcraea son menos claras que para el resto de los géneros.
En relación con los endemismos, las Serranías Meridionales presentan 64 taxa, 34 (53\%) de ellos se consideran endémicos a la provincia. La Sierra Madre Occidental agrupa a 50 taxa, 31 endémicos, que representan el $62 \%$. Las especies determinadas para estas provincias no se hallan todas en los bosques templados, sino que algunas se restringen a los cañones de los ríos, con clima y vegetación más seca que la de los alrededores. El tercer nivel lo ocupa la Altiplanicie, donde crecen 46 taxa, con 15 (33\%) endémicos. La provincia de Baja California tiene 26 taxa, 16 (62\%) de los cuales son endémicos. Estas cuatro provincias tienen la mayoría de las especies de agaváceas, sin embargo, en el Valle de Tehuacán se concentran 20 especies en un área pequeña de un grado por un grado (García-Mendoza, 1995).

\section{Nolinaceae}

La familia Nolinaceae es endémica a Norteamérica y parte de Centroamérica. Hernández (1993) señala que sus cuatro géneros se distribuyen desde el sur y sureste de los Estados Unidos hasta México, Guatemala, Belice y Honduras (Fig. 2). De las aproximadamente 55 especies, México tiene 49, que representan el $89 \%$ de todas las especies conocidas para la familia (Cuadro 1). Las provincias fitogeográficas más ricas son: para Nolina, la Sierra Madre Occidental con 17 taxa, cinco de ellos son endémicos a la misma y representan el 29\%. La única especie de Calibanus crece en la Altiplanicie, provincia en la que también se desarrollan 11 especies de Dasylirion, cinco (45\%) de ellas endémicas a la misma. 




Figura 2. Distribución de la familia Nolinaceae (modificado de Hernández, 1993). 
CuAdro 3. Distribución de los taxa de Nolinaceae por provincia florística, entre paréntesis las endémicas a la provincia.

\begin{tabular}{|c|c|c|c|c|c|}
\hline PROVINCIAS & Calibanus & Dasylirion & Beaucarnea & Nolina & Total \\
\hline California & & & & $3(0)$ & $3(0)$ \\
\hline Baja California & & & & $5(1)$ & $5(1)$ \\
\hline Sierra Madre Occidental & & $5(3)$ & & $12(5)$ & $17(8)$ \\
\hline Altiplanicie & $1(1)$ & $11(5)$ & & $7(3)$ & $19(9)$ \\
\hline Costa Pacífica & & & $3(2)$ & & $3(2)$ \\
\hline Sierra Madre Oriental & & $3(1)$ & & $3(1)$ & $6(2)$ \\
\hline Serranías Meridionales & & $4(2)$ & & $2(1)$ & $6(3)$ \\
\hline Valle de Tehuacán-Cuicatlán & & $2(1)$ & $3(3)$ & $1(0)$ & $6(4)$ \\
\hline Depresión del Balsas & & & $1(1)$ & & $1(1)$ \\
\hline Costa del Golfo de México & & & $1(1)$ & & $1(1)$ \\
\hline Península de Yucatán & & & $2(1)$ & & $2(1)$ \\
\hline
\end{tabular}

Para Beaucarnea el Valle de Tehuacán-Cuicatlán y la Costa Pacífica representan las áreas más ricas en especies (Cuadro 3). Al considerar los cuatro géneros, la Akiplanicie reúne 19 taxa, con nueve (47\%) endémicos y la Sierra Madre Occidental tiene 17 , con ocho (47\%) endémicos.

Algunos géneros (de ambas familias) se han diversificado con mayor profusión en algunas provincias, tal es el caso de Calibanus, Dasylirion, Hesperaloë y Yucca, cuyo centro de riqueza y endemismo se presenta en la Altiplanicie, mientras que para Manfreda, Nolina, Polianthes y Prochnyanthes la Sierra Madre Occidental y las Serranías Meridionales han jugado un papel importante en su evolución. Agave tiene una historia más compleja, pues su diversificación aparentemente ha seguido dos vías; algunos grupos del subgénero Agave se han adaptado y diversificado mejor en las provincias de las zonas áridas como es la península de Baja California, Altiplanicie y Valle de Tehuacán-Cuicatlán, mientras que otros grupos, principalmente del subgénero Littaea, lo han hecho en las provincias montañosas de las Sierras Madres.

En el apéndice se presenta la lista actualizada de los taxa de Agavaceae y Nolinaceae para México, su ubicación por provincia florística y los estados donde se les encuentra. El número total de especies de ambas familias es de 343, y el número total de taxa es de 402. En el caso de Agavaceae, los estados más ricos son: Oaxaca con 52 taxa, Durango y Puebla con 43 , Sonora y Jalisco con 40 , Coahuila con 35 , Chihuahua con 34, San Luis Potosí con 33 y Nuevo León y Zacatecas con 29. Para Nolinaceae, San Luis Potosí tiene 14, Chihuahua y Oaxaca 11, Durango nueve y Puebla y Sonora siete. Al sumar los números de las dos familias, Oaxaca tendría 63 taxa, Durango 52, Puebla 50, San Luis Potosí y Sonora 47, Chihuahua 45, Jalisco 44, Coahuila 39, Nuevo León y Zacatecas 34 e Hidalgo 32 (ver Cuadro 4). Oaxaca es un estado especialmente interesante pues en su territorio confluyen las provincias semiáridas y templadas del norte y sur del país.

\section{FLORAS E INVENTARIOS FLORÍSTICOS}

En México se han realizado cinco Floras Regionales que incluyen las familias Agavaceae y Nolinaceae. La Flora del Desierto Sonorense (Shreve y Wiggins, 1964) y la Flora de Baja California (Wiggins, 1980), incluyen la península de Baja California y la porción costera y semiárida de Sonora. La Flora de Nueva Galicia (McVaugh, 1989) así como las del Valle de México (Galván, 1990) y Flora Mesoamericana (García-Mendoza y Lott, 1994) tienen revisiones taxonómicas recientes para ambas familias. Cabe señalar que Gentry (1978) revisa la familia Agavaceae (sensu lato) para Sonora y en 1978 el género Agave para la península de Baja California. Además se cuenta con cuatro listas de especies, que complementan este conocimiento para algunas regiones del país, tal es el caso del listado florístico realizado para la Flora de Durango (González et al., 1991), para el estado de Querétaro (Arguielles et al., 1991), para la Flora del Valle de Tehuacán-Cuicatlán (Dávila et al., 1993) y para la Flora de Veracruz (Sosa y Gómez-Pompa, 1994) (Fig. 3). Algunos listados más, como los realizados para Tabasco (Cowan, 1983), Quintana Roo (Sousa y Cabrera, 1983) y Chiapas (Breedlove, 1986), han sido actualizados con la aparición del primer volumen publicado de la Flora Mesoamericana que incluye estas familias. El listado de la estación de Biología Chamela, Jalisco (Lott, 1985), quedó superado con la aparición de la Flora Novo-Galiciana (McVaugh, 1989). Por otro lado, Espejo y López (1993) presentan una lista casi completa de las especies de Agavaceae para México y parte de los estados donde crecen.

El conocimiento más preciso sobre la taxonomía y biogeografía de los grupos es cada vez más completo.Trelease (1920) realiza el primer estudio taxonómico en este siglo, para los géneros Agave y Furcraea, considerados bajo Amaryllidaceae, y para los géneros Hesperaloë, Hesperoyucca, Samuela, Yucca, Nolina, Calibanus, Beaucarnea y Dasylirion 


\section{CuAdRO 4. Distribución de Agavaceae y Nolinaceae por estados.}

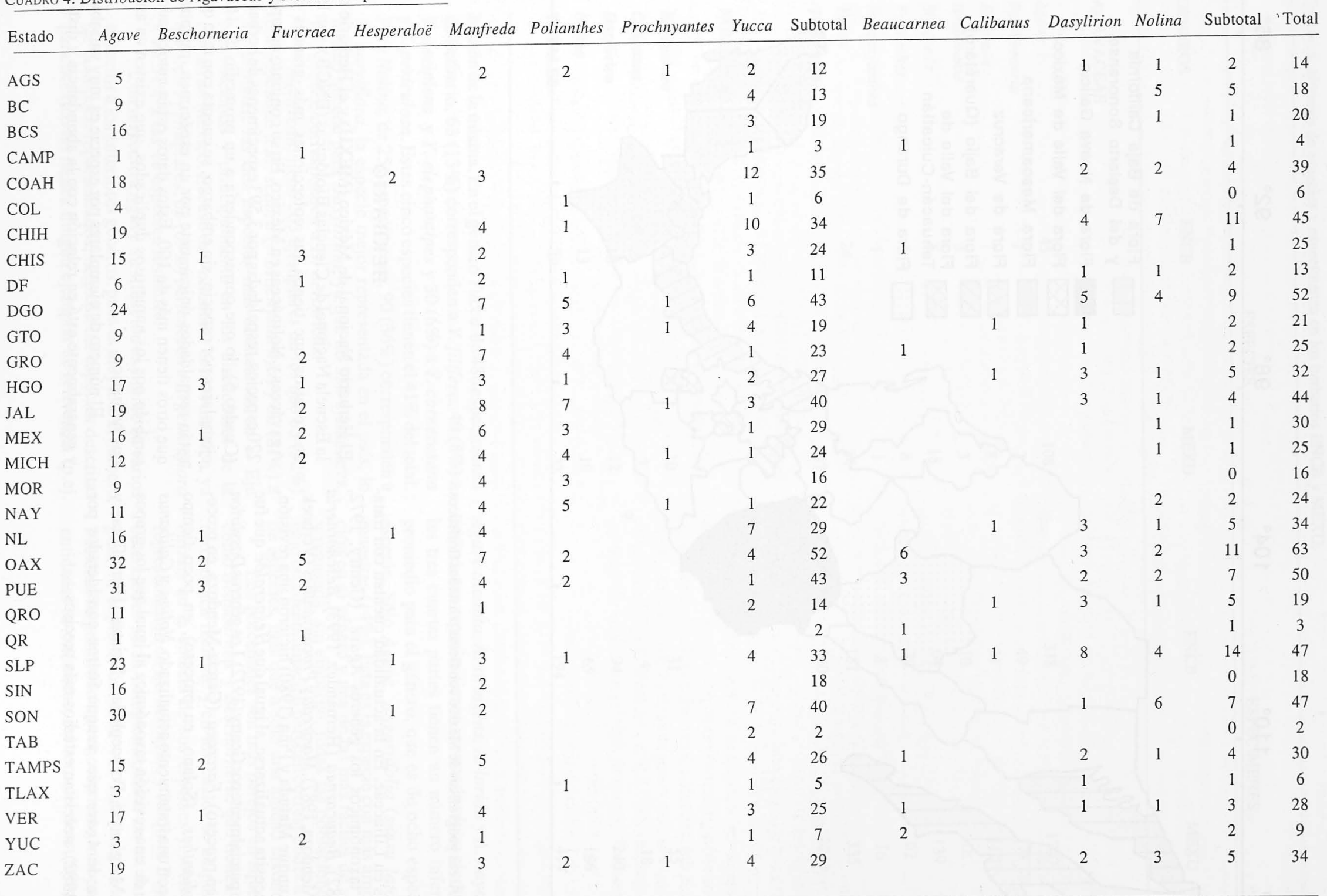




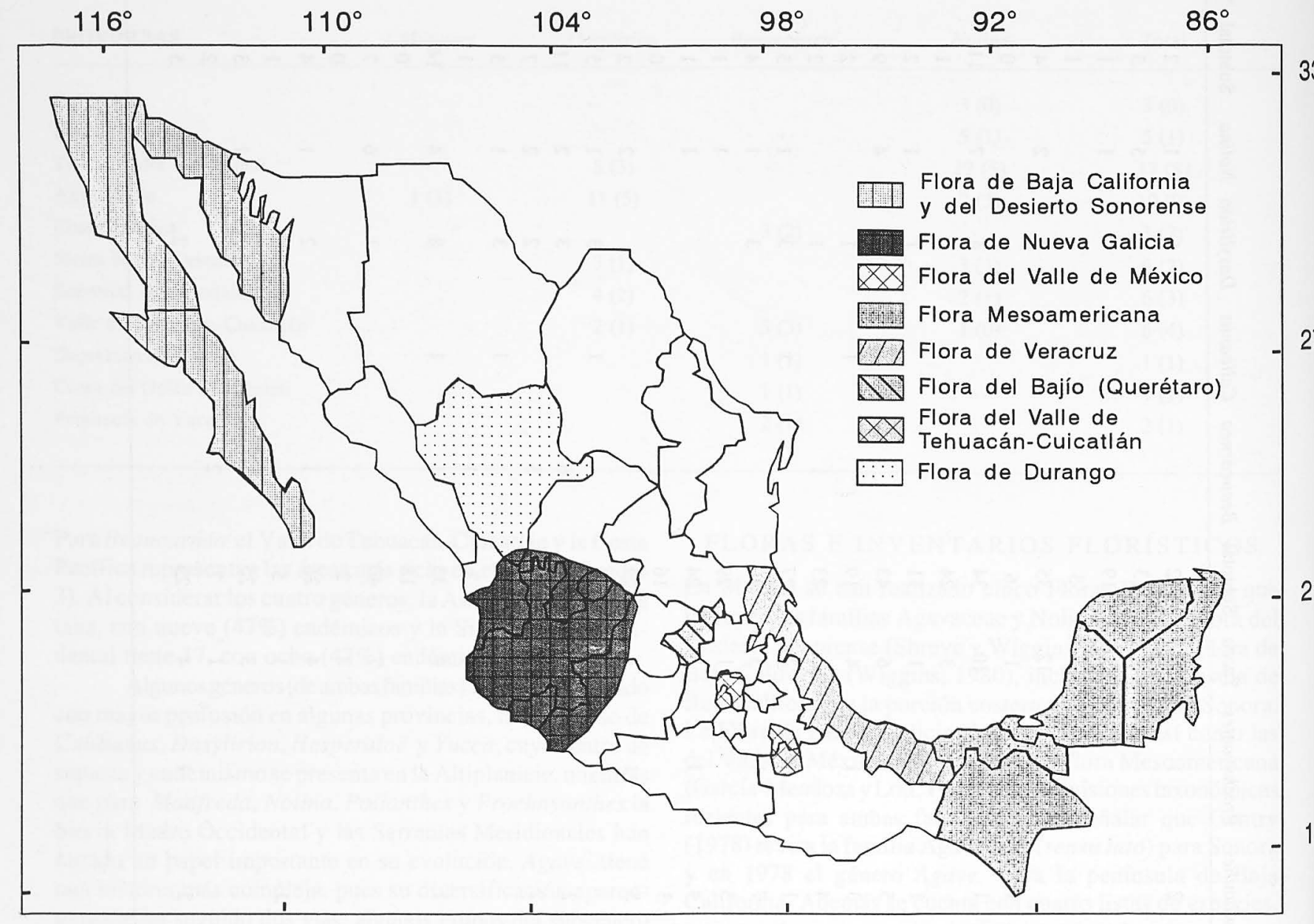

FiguRA 3. Áreas geográficas de México con floras o listados florísticos.

incluidos en Liliaceae. En la actualidad cuentan con tratamientos taxonómicos los géneros Agave (Gentry, 1972, 1978,1982), Beaucarnea (Hernández, 1993), Beschorneria (García-Mendoza, 1987), Manfreday Prochnyanthes (Verhoek, 1975). Aunque Matuda y Piña (1980) hicieron una revisión, Yucca necesita actualizarse, al igual que Hesperaloë que fue estudiado parcialmente porGentry (1972).Los géneros Dasylirion (Bogler, en proceso), Furcraea (García-Mendoza, en proceso) y Polianthes (Solano, en proceso), en poco tiempo contarán con una tratamiento actualizado. Nolina y Calibanus requieren de una revisión taxonómica al igual que los grupos Rigidae, Marginatae, Polycephalae, Striatae, Hiemiflorae y Salmianae de Agave que, aunque fueron considerados por Gentry (1982), necesitan estudios más precisos.

\section{HERB ARIO}

El Herbario Nacional de México (MEXU) y el Herbario de la Escuela Nacional de Ciencias Biológicas (ENCB), son los dos centros que tienen las colecciones más grandes de Agaváceas y Nolináceas en México. En su conjunto agrupan 220 especies, respaldadas por 3,593 especímenes de herbario (Cuadro 5), lo que correspondería a un promedio de 16.3 ejemplares por especie; sin embargo, se cuenta con taxa que están respaldados únicamente por un espécimen, mientras que otros tienen más de 100. Estos datos están aumentados también por los numerosos duplicados, que existen en ambos herbarios.

El número de ejemplares por especie es muy variable, y generalmente está en relación con la abundancia y distri- 
CuAdro 5. Número de especies y especímenes en los herbarios ENCB y MEXU.

GENEROS

ENCB

MEXU

ENCB

MEXU

AGAVACEAE

$\begin{array}{lcccc}\text { Agave } & 45 & 108 & 318 & 1707 \\ \text { Beschorneria } & 7 & 7 & 49 & 171 \\ \text { Furcraea } & 7 & 8 & 31 & 110 \\ \text { Hesperaloe } & 3 & 3 & 10 & 18 \\ \text { Manfreda } & 5 & 18 & 64 & 139 \\ \text { Polianthes } & 6 & 8 & 29 & 62 \\ \text { Prochnyanthes } & 1 & 1 & 8 & 16 \\ \text { Yucca } & 24 & 34 & 151 & 335 \\ \text { TOTALES } & 98 & 187 & 660 & 2240\end{array}$

NOLINACEAE

$\begin{array}{lcccc}\text { Beaucarnea } & 5 & 10 & 31 & 55 \\ \text { Calibanus } & 1 & 1 & 4 & 18 \\ \text { Dasylirion } & 10 & 12 & 94 & 240 \\ \text { Nolina } & 13 & 10 & 65 & 186 \\ \text { TOTALES } & 29 & 33 & 194 & 499\end{array}$

bución de la misma. En el género Yucca de 486 especímenes de herbario, $63(13 \%)$ corresponden a $Y$. filifera, $40(8 \%)$ a $Y$. decipiens y $Y$. elephantipes y $30(6 \%)$ a $Y$. carnerosana y $Y$. periculosa. Estas cinco especies tienen el $41 \%$ del total. Para Nolina, de 251 especímenes, 90 (36\%) corresponden a $N$. parviflora, la especie mejor representada en el país, lo mismo sucede para Dasylirion, ya que de 334 ejemplares, $160(48 \%)$, corresponden a D. acrotriche.

Manfreda tiene 203 ejemplares de los que $65(32 \%)$ corresponden a $M$. scabra (= brachystachya) y $62(31 \%)$ a $M$. pringlei, las dos especies más abundantes en el Eje Neovolcánico y principalmente en los alrededores de la ciudad de México. El caso de Polianthes es más drástico, ya que de 91 ejemplares, $63(69 \%)$ pertenecen a $P$. geminiflora y un $31 \%$ a las 11 especies restantes.

Con respecto a Agave su situación es similar en varios aspectos a los anteriores géneros, pues es muy alto el número de taxa poco representados, ocho de las especies descritas para México no tienen ningún ejemplar de referencia (p.ej.
Agave titanota, A. stringens, A. lurida y A. nayaritensis); el $50 \%$ de las especies tienen menos de cinco ejemplares y casi las tres cuartas partes tienen un número inferior al valor promedio para el género, que es de ocho especímenes por especie. Sólo Agave angustifolia (sensu lato) tiene 240 muestras (14\%). Esta es la especie con más amplia distribución en el género y una de las más fáciles de ser colectada y prensada; sin embargo, todas las poblaciones agrupadas bajo esta denominación parecen corresponder a un complejo de especies, más que a sólo una unidad taxonómica.

Los porcentajes son más parejos en aquellos géneros como Beaucarnea y Beschorneria que tienen una revisión taxonómica reciente y en los cuales se ha puesto especial énfasis en la colecta de sus especies y sobre todo, en su representación en los herbarios y Jardines Botánicos. Estas colecciones serán complementadas en un futuro cercano, pues la exploración cada vez más intensa del territorio nacional ha permitido un conocimiento más profundo de las entidades taxonómicas, así como el descubrimiento de nue- 
vos taxa (García-Mendoza y Palma, 1993; Cházaro, en prensa; Zamudio y Sánchez, en prensa).

\section{CONCLUSIONES}

En México la falta de especímenes de herbario es notoria en casi todas las especies de Agavaceae y Nolinaceae, por lo cual es necesario impulsar su colecta, para tener colecciones regionales más completas. Esto se puede cubrir en parte mediante la impartición de cursos sobre el tema en los herbarios estatales, la elaboración de un manual completo que contenga las técnicas de colecta y prensado, así como las observaciones a realizar sobre las plantas en el campo. Este punto ha sido y es el limitante para efectuar floras y trabajos biogeográficos, aunque el conocimiento mayor sobre la distribución de las especies redundará en beneficio de otras áreas del conocimiento como pueden ser, la conservación, la etnobotánica y la ecología. Los complejos de especies es un tema prioritario a resolver, pues se presentan en varios grupos, principalmente de los géneros Agave, Manfreda, Nolina y Yucca. Es deseable también realizar un mayor número de trabajos en el campo de la biología de la reproducción y de los estudios anatómicos, citológicos y moleculares.

\section{AGRADECIMIENTOS}

Agradecemos a los curadores de los herbarios ENCB y MEXU, el acceso a las colecciones. A la Dra. Karen H. Clary su ayuda en la delimitación geográfica de las Agavaceae. Al Dr. Luis Eguiarte sus comentarios al manuscrito. Al Sr. Felipe Villegas las figs. 1 y 2 y al Biól. Jorge Saldívar y a la Srita. Claudia Vázquez la fig. 3 y las tablas del trabajo.

\section{LITERATURA CITADA}

Argüelles E, Fernández R, Zamudio S. 1991. Flora del Bajío y de regiones adyacentes. Fascículo complementario II. Listado florístico preliminar del estado de Querétaro. Pátzcuaro, Michoacán: Instituto de Ecología, A.C. Centro Regional del Bajío.

Baker JG. 1888. Agaveae. Handbook Amaryllidaceae. London: George Bell and Sons.

Benson L. 1979. Plant classification. Lexington: Heath and Co.

Bentham GD, Hooker JD. 1883. Genera plantarum III. London: Reeve and Co.

Bogler JD, Simpson BB. 1995. A chloroplast DNA study of the Agavaceae. Systematic Botany 20: 191-205.

Breedlove DE. 1986. Listados florísticos de México IV. Flora de Chiapas. México DF: Instituto de Biología, UNAM.

Cave MS. 1964. Cytological observations on some genera of the Agavaceae. Madroño 17: 163-170.

Cowan CP. 1983. Listados florísticos de México I. Flora de Tabasco. México DF: Instituto de Biología, UNAM.

Cronquist A. 1968. The evolution and classification of flowering plants. Boston: Houghton Mifflin.

Cronquist A. 1981. An integrated system of classification of flowering plants. New York: Columbia University Press.

Dahlgren RMT, Clifford HT, Yeo PF. 1985. The families of the monocotyledons. Structure, evolution and taxonomy. Berlín: Springer-Verlag.
Dávila AP, Villaseñor JL, Medina R, Ramírez A, Salinas A, Sánchez-Ken J, Tenorio P. 1993. Listados florísticos de México $X$. Flora del Valle de Tehuacán-Cuicatlán. México DF: Instituto de Biología, UNAM.

Eguiarte LE, Duvall MR, Learn GH, Clegg MT. 1994. The systematic status of the Agavaceae and Nolinaceae and related Asparagales in the Monocotyledons: An analysis based on the $r b c \mathrm{~L}$ gene sequence. Boletín de la Sociedad Botánica de México 54: 35-56.

Endlicher S. 1836-1840. Genera plantarum secundum ordines naturales. Leipzig: Vindobonae.

Endlicher S. 1841. Enchiridion botanicum. Leipzig: Engelman.

Espejo SA, López AR. 1993. Las monocotiledóneas mexicanas, una sinopsisflorística. Parte I. Agavaceae, Alismaceae, Alliaceae, Alstroemeriaceaey Amaryllidaceae. MéxicoDF: Consejo Nacional de la flora de México, A.C. y Universidad Autónoma Metropolitana Iztapalapa.

Galván VR. 1990.Liliaceae, Amaryllidaceae. En:Rzedowski J, Rzedowski GC. (Eds.). Flora fanerogámica del Valle de México. Vol. III: 289-320. Pátzcuaro, Michoacán: Instituto de Ecología, A.C. Centro Regional del Bajío.

García-Mendoza A.1987. Monografía del género Beschorneria Kunth. Agavaceae. Tesis de Maestría. México DF: Facultad de Ciencias, UNAM.

García-Mendoza A, Lott E. 1994. Agavaceae. En: Davidse G, Sousa M, Chater AO. (Eds.).Flora Mesoamericana. Vol. 6: 35-47. México DF: UNAM.

García-Mendoza A.1995. Riqueza y endemismos de la familia Agavaceae en México. En: Linares E, Dávila P, Chiang F, Bye R, Elias T. (Eds.). Conservación de plantas en peligro de extinción: $\mathrm{Di}$ ferentes enfoques. 59-83. México DF: UNAM.

García-Mendoza A, Palma F. 1993. Una nueva especie de Agave (Agavaceae, subgénero Agave) de Oaxaca y Chiapas, México. Sida 15: 565-568.

Gentry HS. 1972. The Agave family in Sonora. Washington DC: Agricultural Research Service U.S. Department of Agriculture. Agriculture Handbook 399.

Gentry HS. 1978. The Agaves of Baja California. San Francisco: Occasional Papers of the California Academy of Sciences 130.

Gentry HS. 1982. Agaves of Continental North America. Tucson: University Arizona Press.

Gómez-Pompa A. 1963. El género Agave. Cactáceas y Suculentas Mexicanas 8: 3-25.

Gómez-Pompa A, Villalobos-Pietrini R, Chimal A. 1971. Studies in the Agavaceae. I. Chromosome morphology and number of seven species. Madroño 21: 208-221.

González EM, González ES, Herrera Y. 1991. Listados florísticos de México IX. Flora de Durango. México DF: Instituto de Biología, UNAM.

Granick E. 1944. A karyosistematic study of the genus Agave. American Journal of Botany 31: 283-298.

Hernández SL. 1993. Cladistic analysis of the American genera of Asparagales and the systematic study of Beaucarnea (Nolinaceae) and Hemiphylacus (Hyacinthaceae). $\mathrm{PhD}$ thesis. Austin, USA: The University of Texas.

Hutchinson J. 1934. The families of flowering plants. Vol. II. Monocotyledons. London: The Macmillan Co.

Hutchinson J. 1959. The families of flowering plants. Vol. II. Monocotyledons. Second ed. Oxford: Oxford University Press.

Lawrence GHM. 1951. Taxonomy of vascular plants. New York: The Macmillan Co.

Lotsy A. 1911. Agavaceae. Vorträge, ÜberBotanische Stammesgeschichte. Cormophyta Siphonogamia. Band 3: 806. Jena: G. Fischer. 
Lott EJ. 1985. Listados florísticos de México III. La estación de biología Chamela, Jalisco. México DF: Instituto de Biología, UNAM.

Matuda E, Piña LI. 1980. Las plantas mexicanas del género Yucca. México DF: Colección Misc. Edo. de México-Laboratorios Nacionales de Fomento Industrial.

McKelvey DS, Sax K. 1933. Taxonomical and cytological relationships of Yucca and Agave. Journal of the Arnold Arboretum 14: 76-81.

McVaugh R. 1989. Liliaceae. En: Anderson WR. (Ed.). Flora NovoGaliciana. A descriptive account of the vascular plants of Western Mexico. Vol. 15: 120-293. Ann Arbor: The University of Michigan Herbarium.

Pax F. 1887. Amaryllidaceae. En: Engler A, Prantl K. (Eds.). Die Natürlichen Pflanzenfamilien. Leipzig, Verlag.

Pax F, Hoffmann K. 1930. Amaryllidaceae. En: Engler A, Prantl K. (Eds.). Die Natürlichen Pflanzenfamilien. Leipzig: Engelmann.

Porter CL. 1967. Taxonomy offlowering plants. San Francisco: Freeman and $\mathrm{Co}$.

Rzedowski J. 1978. Vegetación de México. México DF: Limusa.

Salisbury RA. 1866. The genera of plants. London: John Van Voorst.

Satô D. 1935. Aıralysis of the karyotypes in Yucca, Agave and related genera with special reference to the phylogenetic significance. Japanese Journal of Genetics 11: 273-278.

Satô D. 1942. Karyotipe alteration and phylogeny in the Liliaceae and allied families. Japanese Journal of Botany 12: 57-161.

Shreve F, Wiggins LI. 1964. Vegetation and Flora of the Sonoran Desert. Stanford, Stanford University Press.
Sosa V, Gómez-Pompa A.1994. Flora de Veracruz, Lista florística. Fascículo 82. Xalapa, Veracruz: Instituto de Ecología, A.C., University of California, Riverside, CA.

Sousa SM, Cabrera FE. 1983. Listados florísticos de México II. Flora de Quintana Roo . México DF: Instituto de Biología, UNAM.

Speiers DC. 1979. Yucca glauca (Agavaceae) in Western Canada. National Cactus and Succulent Journal 34: 59.

Takhtajan AL. 1980. Outline of the classification of flowering plants (Magnoliophyta). Botanical Review 46: 225-359.

Thorne RF. 1968. Synopsis of putatively phylogenetic classification of flowering plants. Aliso 6: 51-57.

Trelease W. 1920. Liliaceae, Amaryllidaceae. En: Standley PC. (Ed.). Trees and shrubs of Mexico. Contributions from the United States National Herbarium 23: 86-142.

Verhoek WS. 1975. A study of the tribe Poliantheae (including Manfreda and revisions of Manfreda and Prochnyanthes (Agavaceae). $\mathrm{PhD}$ thesis. Ithaca, New York: Cornell University, Whitaker JW. 1934. Chromosome constitution in certain Monocotyledons. Journal of the Arnold Arboretum 31: 283-289.

Wiggins LI. 1980. Flora of Baja California. Stanford: Stanford University Press.

Wunderlich R. 1950. Die Agavaceae Hutchinsons im lichte ihrer Embryologie, ihres Gynözeum-, Staubblatt- und Blattbaues. En: Geitler L. (Ed.). Österreichische Botanische Zeitschrift. Viena: Springer-Verlag.

\section{APÉNDICE}

Lista de las especies ce Agavaceae y Nolinaceae para México, la(s) provincia(s) florística(s) y estados donde crecen. Provincias: 0. California. 1. Baja California. 2. Planicie Costera del Noroeste. 3. Sierra Madre Occidental. 4. Altiplanicie. 5. Planicie Costera del Noreste. 6. Costa Pacífica. 7. Sierra Madre Oriental. 8. Serranías Meridionales. 9. Valle de TehuacánCuicatlán. 10. Depresión del Balsas. 11. Costa del Golfo de México. 12. Serranías Transístmicas. 13. Península de Yucatán. 14. Islas de Revillagigedo. 15. Especies no conocidas en estado silvestre, pero que se supone son nativas de México. 16. Cultivar.

* Especies endémicas a la provincia.

Clave de los estados: AGS (Aguascalientes), BC (Baja California), BCS (Baja California Sur), CAMP (Campeche), COAH (Coahuila), COL (Colima), CHIH (Chihuahua), CHIS (Chiapas), D.F. (Distrito Federal), DGO (Durango), GTO (Guanajuato), GRO (Guerrero), HGO (Hidalgo), JAL (Jalisco), MEX (estado de México), MICH (Michoacán), MOR (Morelos), NAY (Nayarit), NL (Nuevo León), OAX (Oaxaca), PUE (Puebla), QRO (Querétaro), QR (Quintana Roo), SLP (San Luis Potosí), SIN (Sinaloa), SON (Sonora), TAB (Tabasco), TAMPS (Tamaulipas), TLAX (Tlaxcala), VER (Veracruz), YUC (Yucatán) y ZAC (Zacatecas).

\section{A GAVACEAE}

\section{AGAVE}

Agave aktites H. Gentry (2)*. SIN, SON.

Agave albomarginata $\mathrm{H}$. Gentry (15).

Agave americana L. var. americana $(4,16)$. BCS, CHIH, COAH, DGO, HGO, GTO, JAL, MEX MOR, NL, OAX, PUE, QRO, SLP, TAMPS, VER, ZAC.

Agave americana L. var. expansa (Jacobi) H. Gentry (16). JAL, SON. 
Agave americana L. var. marginata Hort. (16).

Agave americana L. var. oaxacensis $\mathrm{H}$. Gentry $(8,16)$. OAX, SLP.

Agave americana L. var. picta (Salm-Dyck) A. Terrac. (16).

Agave americana L. subsp. protamericana $\mathrm{H}$. Gentry (7)*. HGO, NL, SLP, TAMPS.

Agave angustiarum Trel. (10)*. GRO, MICH, MEX, MOR, OAX, PUE.

Agave angustifolia Haw. var. angustifolia $(2,3,4,6,7,8,10,11,13,14)$. AGS, CAMP, CHIH, CHIS, DGO, GRO, JAL, MICH, MEX, MOR, NAY, NL, OAX, PUE, Q.R., SIN, SON, TAMPS, TLAX, VER, YUC, ZAC.

Agave angustifolia Haw. var. deweyana (Trel.) H. Gentry (16). TAMPS, VER.

Agave angustifolia Haw. var. marginata Hort. (16).

Agave angustifolia Haw. var. rubescens (Salm-Dyck) H. Gentry (6,10). DGO, GRO, JAL, OAX, PUE, SIN, SON, VER.

Agave angustifolia Haw. var. sargentii Trel. (10)*. MOR, PUE.

Agave applanata K. Koch ex Jacobi $(3,4,7,8)$. CHIH, DGO, GTO, HGO, MEX, OAX, PUE, QRO, VER, ZAC.

Agave asperrima Jacobi subsp. asperrima (4)*. CHIH, COAH, DGO, NL, ZAC.

Agave asperrima Jacobi subsp. maderensis (H. Gentry) Ullrich (7)*. COAH.

Agave asperrima Jacobi subsp. potosiensis (H. Gentry) Ullrich (4)*. QRO, NL, SLP.

Agave asperrima Jacobi subsp. zarcensis (H. Gentry) Ullrich (4)*. DGO.

Agave atrovirens Karw. ex Salm-Dyck var. atrovirens (8)*. OAX, PUE, VER.

Agave atrovirens Karw. ex Salm-Dyck var. mirabilis (Trel.) Gentry (8)*. PUE, VER.

Agave attenuata Salm-Dyck (8)*. MEX, MICH, JAL.

Agave aurea Brandegee (1)*. BCS.

Agave avellanidens Trel. (1)*. BCS.

Agave bakeri Hook. f. (8)* MEX.

Agave bovicornuta H. Gentry (3)* CHIH, SIN, SON.

Agave bracteosa $\mathrm{S}$. Watson ex Engelm. (7)*. COAH, NL.

Agave breedlovei H. Gentry (12)*. CHIS.

Agave cantala Roxb. var. acuispina (Trel.) Gentry (16). CHIS.

Agave capensis H. Gentry (1)*. BCS.

Agave celsii Hook. var. albicans (Jacobi) H. Gentry (7)*. HGO.

Agave celsii Hook. var. celsii (7)*. HGO, PUE, NL, QRO, SLP, TAMPS.

Agave cerulata Trel. subsp. cerulata (1)*. BC, BCS.

Agave cerulata Trel. subsp. dentiens (Trel.) H. Gentry (1)*. BC.

Agave cerulata Trel. subsp. nelsonii (Trel.) H. Gentry $(0,1)$. BC.

Agave cerulata Trel. subsp. subcerulata H. Gentry (1)*. BCS.

Agave colimana H. Gentry (6)*. COL. JAL, MICH.

Agave colorata H. Gentry (2)*. SIN, SON.

Agave congesta $\mathrm{H}$. Gentry (12)*. CHIS.

Agave cupreata Trel. \& Berger (10)*. GRO, MICH.

Agave chiapensis Jacobi (12)*. CHIS.

Agave chrysoglossa Johnston (2)*. SON.

Agave dasylirioides Jacobi \& Bouché $(7,8)$. MOR, SLP.

Agave datylio C. Weber var. datylio (1)*. BCS

Agave datylio C. Weber var. vexans (Trel.) I.M. Johnston (1)*. BCS.

Agave deserti Engelm. subsp. deserti $(0,1)$. BC.

Agave deserti Engelm. subsp. pringlei (Engelm. ex Baker) H. Gentry $(0,1)$. BC.

Agave deserti Engelm. subsp. simplex H. Gentry (2). SON.

Agave desmettiana Jacobi (15).

Agaie difformis Berger (4,7). HGO, SLP

Agave durangensis $\mathrm{H}$. Gentry (3)*. DGO, ZAC.

Agave elleemettiana Jacobi (8)* MEX.

Agave ensifera Jacobi (15).

Agave felgeri $\mathrm{H}$. Gentry (2)*. SON.

Agave filifera Salm-Dyck $(4,7,10)$. AGS, GTO, HGO, MEX, MICH, QRO, SLP, VER.

Agave flexispina Trel. (3,4). CHIH, DGO, ZAC.

Agave fortiflora $\mathrm{H}$. Gentry (2)*. SON.

Agave fourcroydes Lem. (16). NL, OAX, TAMPS, YUC. 
Agave funkiana K. Koch \& Bouché (4)*. NL, SLP, TAMPS.

Agave geminiflora (Tagliabue) Ker Gawler (3)*. NAY, SIN.

Agave ghiesbreghtii Lem. ex Jacobi $(6,8,9)$. CHIS, GRO, MEX, OAX, PUE.

Agave gigantensis H. Gentry (1)*. BCS.

Agave glomeruliflora (Engelm.) A. Berger (4). COAH.

Agave gracilipes Trel. (4). CHIH.

Agave guadalajarana Trel. (8)*. JAL.

Agave guiengola $\mathrm{H}$. Gentry (6)*. OAX.

Agave gypsophila $\mathrm{H}$. Gentry (10)*. COL. GRO, JAL.

Agave havardiana Trel. (4). $\mathrm{CHIH}, \mathrm{COAH}$.

Agave hiemiflora $\mathrm{H}$. Gentry (12). CHIS.

Agave hookeri Jacobi $(8,10)$. GRO, JAL. MICH.

Agave horrida Lem. ex Jacobi subsp. horrida (8)*. MEX, MOR, SLP.

Agave horrida Lem. ex Jacobi subsp. perotensis Ullrich (8)*. PUE, VER.

Agave impressa H. Gentry (6)*. NAY, SIN.

Agave inaequidens K. Koch subsp. barrancensis H. Gentry (3)*. DGO.

Agave inaequidens K. Koch subsp. inaequidens (8)*. D.F., HGO, JAL, MEX, MICH, MOR, PUE.

Agave isthmensis García-Mendoza \& Palma (6)*. CHIS, OAX.

Agave jaiboli $\mathrm{H}$. Gentry (3)*. SON.

Agave karwinskii Zucc. $(8,9,11)$. OAX. PUE, VER.

Agave kerchovei Lem. $(8,9)$. HGO, OAX, PUE.

Agave kewensis Jacobi (6)*. CHIS.

Agave kirchneriana Berger (10)*. GRO.

Agave lechuguilla Torrey (4). CHIH, COAH, D.F., DGO, HGO, MEX, NL, QRO, SLP, TAMPS, ZAC.

Agave lophantha Schiede (7)*. COAH, NL, PUE, SLP, TAMPS, VER.

Agave lurida Aiton (9)*. OAX.

Agave macroacantha Zucc. (9)*. OAX, PUE.

Agave macroculmis Tod. (7,8). COAH, D.F., DGO, HGO, NL, PUE, SLP, TAMPS, ZAC.

Agave mapisaga Trel. var. mapisaga (16). D.F., HGO, MEX, MOR, OAX, PUE, QRO, SLP, TAMPS, TLAX, VER, ZAC.

Agave margaritae Brandegee (1)*. BCS.

Agave marmorata Roezl $(8,9)$. OAX, PUE.

Agave maximiliana Baker var. katharinae (A. Berger) H. Gentry $(3,8)$. COL, DGO, JAL, NAY, SIN.

Agave maximiliana Baker var. maximiliana $(3,8)$. DGO, JAL, NAY, ZAC.

Agave moranii $\mathrm{H}$. Gentry $(0,1)$. BC.

Agave multifilifera $\mathrm{H}$. Gentry (3)*. CHIH, DGO, SIN, SON.

Agave murpheyi F. Gibson (2). SON.

Agave nayaritensis H. Gentry (6)*. NAY.

Agave neomexicana Wooton \& Standley (4). COAH.

Agave nizandensis Cutak (6)*. OAX.

Agave obscura Schiede (8)*. OAX, PUE, SLP, TAMPS, VER.

Agave ocahui $\mathrm{H}$. Gentry var. longifolia $\mathrm{H}$. Gentry (3)*. SON.

Agave ocahui $\mathrm{H}$. Gentry var. ocahui (3)*. SON.

Agave ornithobroma H. Gentry (3)*. NAY, SIN.

Agave oroensis $\mathrm{H}$. Gentry (15). ZAC.

Agave pachycentra Trel. (12). CHIS, OAX.

Agave palmeri Engelm. (2,3). CHIH, SON.

Agave parrasana A. Berger (7)*. COAH.

Agave parryi Engelm. var. huachucensis (Baker) Little in Benson (3)*. SON.

Agave parryi Engelm. var. parryi $(3,4)$. CHIH, DGO, GTO.

Agave parryi Engelm. var. truncata $\mathrm{H}$. Gentry (3)*. DGO, ZAC.

Agave parviflora Torrey subsp. flexiflora $\mathrm{H}$. Gentry (3)*. SON.

Agave parviflora Torrey subsp. parviflora (3). SON.

Agave peacockii Croucher $(4,9)$. HGO, OAX, PUE.

Agave pedunculifera Trel. $(3,8)$. GRO, JAL, MICH, NAY, OAX, SIN.

Agave pelona $\mathrm{H}$. Gentry (2)*. SON. 
Agave pendula Schnitts. (7,11,12). CHIS, VER.

Agave polianthiflora H. Gentry (3)*. CHIH, SON.

Agave polyacantha Haw. (7,8). OAX, SLP, TAMPS, VER.

Agave potatorum Zucc. $(8,9)$. OAX, PUE.

Agave potrerana Trel. $(3,4)$. CHIH, COAH, ZAC.

Agave promontorii Trel. (1)*. BCS.

Agave pumila Smet ex Baker (15).

Agave pygmaea $\mathrm{H}$. Gentry (12)*. CHIS.

Agave rhodacantha Trel. (3,8,9). JAL, NAY, OAX, PUE, SIN, SON.

Agave salmiana Otto ex Salm-Dyck var. angustifolia A. Berger (16). D.F., MEX, PUE.

Agave salmiana Otto ex Salm-Dyck subsp. crassispina (Trel.) H. Gentry $(4,8,9)$. COAH, DGO, GTO, HGO, MEX, PUE, SLP, ZAC.

Agave salmiana Otto ex Salm-Dyck var. ferox (K. Koch) H. Gentry (8,9). OAX, PUE.

Agave salmiana Otto ex Salm-Dyck var. salmiana $(4,8,9,16)$. AGS, COAH, COL, D.F., DGO, GTO, HGO, JAL, MEX, MICH, MOR, OAX, PUE, QRO, SLP, TLAX, VER, ZAC.

Agave scaposa $\mathrm{H}$. Gentry (8)*. OAX, PUE.

Agave schidigera Lem. (3,6,8). AGS, CHIH, DGO, GTO, JAL, MICH, NAY, SIN, SLP, ZAC.

Agave schottii Engelm. var. schottii $(2,3)$. SON.

Agave sebastiana E. Greene (1)*. BC, BCS.

Agave seemanniana Jacobi $(8,12)$. CHIS, OAX

Agave shawii Engelm. subsp. goldmaniana (Trel.) H. Gentry (1)*. BC.

Agave shawii Engelm. subsp. shawii $(0,1)$. BC.

Agave shrevei $\mathrm{H}$. Gentry subsp. magna $\mathrm{H}$. Gentry (3)* CHIH, SIN, SON.

Agave shrevei $\mathrm{H}$. Gentry subsp. matapensis H. Gentry (3)*. SON.

Agave shrevei $\mathrm{H}$. Gentry subsp. shrevei (3)*. CHIH, SON.

Agave sisalana Perrine (16). CHIS.

Agave sobria Brandegee subsp. frailensis $\mathrm{H}$. Gentry (1)*. BCS.

Agave sobria Brandegee subsp. roseana (Trel.) H. Gentry (1)*. BCS.

Agave sobria Brandegee subsp. sobria (1)*. BCS.

Agave striata Zucc. subsp. falcata (Engelm.) H. Gentry (4)*. COAH, DGO, NL, SLP, ZAC.

Agave striata Zucc. subsp. striata (4,5,7). COAH, DGO, HGO, NL, PUE, QRO, SLP, TAMPS, ZAC.

Agave stricta Salm-Dyck (9)*. PUE.

Agave stringens Trel. (8)*. JAL.

Agave subsimplex Trel. (2)*. SON.

Agave tequilana Weber (16). GTO, JAL, MICH, NAY.

Agave terracianoi $\operatorname{Pax}(15)$. YUC.

Agave titanota $\mathrm{H}$. Gentry (9)*. OAX.

Agave triangularis Jacobi (9)*. OAX, PUE.

Agave victoriae-reginae T. Moore (7)*. COAH, DGO, NL.

Agave vilmoriniana A. Berger (3)*. AGS, CHIH, DGO, JAL, SIN, SON, ZAC.

Agave vizcainoensis $\mathrm{H}$. Gentry (1)*. BCS.

Agave warelliana Baker (12)*. CHIS.

Agave weberi Cels ex Poisson (15). COAH, NL, SLP.

Agave wocomahi H. Gentry (3)*. CHIH, DGO, SIN, SON.

Agave xylonacantha Salm-Dyck $(4,5)$. GTO, HGO, QRO, SLP, TAMPS.

Agave yuccifolia DC. (15).

Agave zebra H. Gentry (2)*. SON.

Agave sp. nov. Cházaro (11)*. VER.

Agave sp. nov. García-Mendoza (8)*. OAX.

Agave sp. nov. Zamudio \& Sánchez (7)*. QRO.

\section{BESCHORNERIA}

Beschorneria albiflora Matuda (8,12). CHIS, OAX.

Beschorneria calcicola García-Mendoza $(8,9)$. OAX, PUE.

Beschorneria rigida Rose (7,9). GTO, PUE, SLP, TAMPS. 
Beschorneria septentrionalis García-Mendoza (7)*. NL, TAMPS.

Beschorneria tubiflora (Kunth \& Bouché) Kunth (7)*. HGO.

Beschorneria wrightii Hooker (8)*. MEX.

Beschorneria yuccoides K. Koch subsp. dekosteriana (K. Koch) García-Mendoza (7)*. HGO, PUE, VER. Beschorneria yuccoides K. Koch subsp. yuccoides (7)*. HGO.

\section{FURCRAEA}

Furcraea bedinghausii K. Koch (8)*. D.F., HGO, JAL, MEX, MICH.

Furcraea cabuya Trel. (16). YUC.

Furcraea cahum Trel. (13)*. CAMP, Q.R., YUC.

Furcraea guatemalensis Trel. (12). CHIS, OAX.

Furcraea guerrerensis Matuda $(6,8,10)$. GRO. JAL, MICH, OAX.

Furcraea longaeva Karw. \& Zucc. (8)*. GRO, OAX, PUE.

Furcraea macdougallii Matuda (6,9). OAX, PUE.

Furcraea pubescens Todaro (8)*. MEX.

Furcraea quicheensis Trel. (12). CHIS.

Furcraea samalana Trel. (12). CHIS.

Furcraea sp. nov. García-Mendoza (6)*. OAX.

\section{HESPERALOË}

Hesperaloë funifera K. Koch (4). COAH, NL, SLP.

Hesperaloë nocturna Gentry (2)*. SON.

Hesperaloë parviflora (Torrey) Coulter (4). COAH.

\section{MANFREDA}

Manfreda brunnea (S. Watson) Rose (4)*. CHIH, COAH, DGO.

Manfreda chamelensis Lott \& Verhoek (6)*. GRO, JAL.

Manfreda elongata Rose (3)*. DGO, JAL, NAY.

Manfreda guerrerensis Matuda (8)*. GRO.

Manfreda guttata (Jacobi \& Bouché) Rose $(3,4)$. AGS, CHIH, DGO, JAL, QRO, SLP, ZAC.

Manfreda hauniensis (B. Petersen) Verhoek (8)*. GRO, MEX, MOR, OAX.

Manfreda involuta McVaugh (3)*. JAL.

Manfreda jaliscana Rose $(3,8)$. DGO, JAL, MICH, NAY, SIN, SON.

Manfreda langlassei André (8)*. GRO.

Manfreda longibracteata Verhoek (8)*. MICH.

Manfreda longiflora (Rose) Verhoek (5). NL, TAMPS.

Manfreda maculata (C. Martius) Rose (8)*. GRO, MEX, OAX.

Manfreda maculosa (Hook.) Rose (5). COAH, NL, TAMPS.

Manfreda nanchititlensis Matuda (8)*. MEX.

Manfreda planifolia (S. Watson) Rose (3)*. CHIH, SON.

Manfreda potosina (Robinson \& Greenman) Rose (4)*. COAH, DGO, SLP, ZAC.

Manfreda pringlei Rose (8)*. D.F., HGO, JAL, MICH, MEX, MOR, OAX, PUE, VER.

Manfreda pubescens (Regel \& Ortgies) Verhoek (8)*. CHIS, GRO, MOR, OAX.

Manfreda revoluta (Klotzch) Rose (8)*. MEX.

Manfreda rubescens Rose (3)*. JAL, NAY, VER.

Manfreda scabra (Ortega) McVaugh (7,8,11,12). AGS, CHIS, D.F., DGO, GRO, GTO, HGO, JAL, MEX, MICH, MOR, NAY, OAX, PUE, VER.

Manfreda sileri Verhoek (5). TAMPS.

Manfreda singuliflora (S. Watson) Rose (3)*. CHIH, DGO, SIN, ZAC.

Manfreda variegata (Jacobi) Rose (5,7). HGO, NL, PUE, SLP, TAMPS, VER, YUC.

Manfreda virginica (L.) Rose (4). NL, TAMPS.

Manfreda sp. nov. García-Mendoza (8)*. OAX.

Manfreda sp. (9). OAX, PUE. 


\section{POLIANTHES}

Polianthes densiflora (Robinson \& Fern.) Shinners (3)*. CHIH.

Polianthes durangensis Rose (3)*. DGO, NAY.

Polianthes elongata Rose (8)*. GRO.

Polianthes geminiflora (Llave) Rose var. clivicola McVaugh (8)*. JAL, MICH.

Polianthes geminiflora (Llave) Rose var. geminiflora (3,8). D.F., GRO, GTO, HGO, JAL, MEX, MICH, MOR, NAY, OAX, PUE, TLAX.

Polianthes geminiflora (Llave) Rose var. graminifolia (Rose) McVaugh (3,8). AGS, GTO, JAL, ZAC.

Polianthes howardii Verhoek (8)*. COL, JAL.

Polianthes longiflora Rose (8)*. DGO, JAL.

Polianthes michoacana M. Cedano, Delgadillo \& Enciso (8)*. MICH.

Polianthes montana Rose (3)*. NAY.

Polianthes nelsonii Rose (3)*. DGO.

Polianthes palustris Rose (3)*. DGO, NAY.

Polianthes platyphylla Rose (3)*. DGO, JAL, ZAC. MOR, NAY, SLP

Polianthes pringlei Rose $(3,8)$. AGS, GRO, GTO, JAL, MEX, MICH.

Polianthes tuberosa L. (16).

Polianthes tuberosa L.f. plena Moldenke (16). GRO, MEX, MOR, OAX, PUE.

\section{PROCHNYANTHES}

Prochnyanthes mexicana (Zucc.) Rose (3,8). AGS, DGO, GTO, JAL, MICH, NAY, ZAC.

\section{YUCCA}

Yucca aloifolia L. $(6,16)$. CHIS, GRO, OAX.

Yucca arizonica McKelvey (2). SON.

Yucca baccata Torrey var. baccata (4). CHIH.

Yucca brevifolia Engelm. (2). SON.

Yucca carnerosana (Trel.) McKelvey (4). CHIH, COAH, DGO, NL, SLP, TAMPS, ZAC.

Yucca coahuilensis Matuda \& Piña (4)*. COAH.

Yucca decipiens Trel. (4)*. AGS, DGO, GTO, JAL, SLP, ZAC.

Yucca elata (Engelm.) Engelm. (4). CHIH, COAH, SON.

Yucca elephantipes Regel $(11,16)$. CHIS, TAB, VER, YUC.

Yucca endlichiana Trel. (4)*. COAH.

Yucca filifera Chabaud (4)*. AGS, CHIH, COAH, D.F., DGO, GTO, HGO, JAL, MEX, MICH, NL, QRO, SLP, TAMPS, ZAC. Yucca grandiflora H. Gentry (3)*. SON.

Yucca jaliscensis (Trel.) Trel. (8)*. COL, GTO, JAL, NAY.

Yucca lacandonica Gómez-Pompa \& Valdés (11)*. CAMP, CHIS, OAX, TAB, VER.

Yucca linearifolia Clary (4)*. COAH, NL.

Yucca madrensis H. Gentry (3)*. CHIH, SON.

Yucca periculosa Baker (9)*. OAX, PUE, TLAX, VER.

Yucca potosina Rzedowski (4)*. SLP.

Yucca queretaroensis Piña (4)*. GTO, HGO, QRO.

Yucca reverchoni Trel. (4). COAH.

Yucca rigida (Engelm.) Trel. (4)*. CHIH, COAH, DGO, NL, ZAC.

Yucca rostrata (Engelm.) Trel. (4). $\mathrm{CHIH}, \mathrm{COAH}$.

Yucca schidigera Roezl ex Ortgies $(0,1)$. BC, SON.

Yucca schottii Engelm. (4). CHIH, SON.

Yucca thompsoniana Trel. (4). CHIH, COAH, NL.

Yucca torreyi Shafer (4). CHIH, COAH, DGO, NL, TAMPS.

Yucca treculeana Carr. $(4,5)$. COAH, DGO, NL, TAMPS.

Yucca valida Brandegee $(0,1)$. BC, BCS.

Yucca whipplei Torrey subsp. eremica Epling \& Haines $(0,1)$. BC, BCS.

Yucca whipplei Torrey subsp. whipplei $(0,1)$. BC, BCS.

Yucca sp. nov. García-Mendoza. (8)*. OAX. 


\section{BEAUCARNEA}

NOLINACEAE

Beaucarnea goldmanii Rose (6). CHIS.

Beaucarnea gracilis Lem. (9)*. OAX, PUE.

Beaucarnea hiriartiae L. Hernández (10)*. GRO.

Beaucarnea pliabilis (Baker) Rose var. petenensis (Lundell) L. Hernández (13). CAMP. Q.R., YUC.

Beaucarnea pliabilis (Baker) Rose var. pliabilis (13)*. YUC.

Beaucarnea purpusii Rose (9)*. OAX, PUE.

Beaucarnea recurvata Lem. (11)*. OAX, PUE, SLP, TAMPS, VER.

Beaucarnea stricta Lem. (9)*. OAX.

Beaucarnea sp. nov. L. Hernández (6)*. OAX.

Beaucarnea sp. nov. L. Hernández (6)*. OAX.

\section{CALIBANUS}

Calibanus hookeri (Lem.) Trel. (4)*. GTO, HGO, NL, QRO, SLP.

\section{DAS YLIRION}

Dasylirion acrotriche (Schiede ex. Schult.) Otto $(4,8,9)$. AGS, D.F., DGO, GRO, GTO, HGO, JAL, OAX, PUE, QRO, SLP, TLAX, VER, ZAC.

Dasylirion berlandieri S. Watson (7)*. NL, TAMPS.

Dasylirion cedrosanum Trel. (4)*. COAH, ZAC.

Dasylirion durangense Trel. (3)*. CHIH, DGO,

Dasylirion graminifolium (4)*. SLP.

Dasylirion leiophyllum Engelm. ex Trel. (4). CHIH,

Dasylirion longissimum (4,7). HGO, NL, QRO, SLP, TAMPS.

Dasylirion longystilum MacBride (4)*. QRO, SLP.

Dasylirion lucidum Rose (9)*. OAX, PUE.

Dasylirion palaciosii Rzedowski (4)*. SLP.

Dasylirion parryanum Trel. (4)*. SLP.

Dasylirion serratifolium (Karw. ex Schult.) Zucc. (8)*. OAX.

Dasylirion simplex Trel. (3)*. DGO.

Dasylirion texanum Scheele (4). COAH, DGO, NL, SLP.

Dasylirion wheeleri S. Watson ex Rothrock $(3,4)$. CHIH, DGO, SON.

Dasylirion sp.1 (8)*. JAL.

Dasylirion sp.2 $(3,8)$. JAL.

Dasylirion sp. nov. Bogler (3)*. CHIH.

Dasylirion sp. nov. Bogler (4,7). HGO, SLP.

\section{NOLINA}

Nolina affinis Trel. (3)*. CHIH.

Nolina beldingii Trel. (1). BC, BCS.

Nolina bigelovi (Torr.) S. Watson $(0,1,3)$. BC, SON.

Nolina cespitifera Trel. (4)*. COAH, NL.

Nolina compacta S. Watson (3). CHIH, SON.

Nolina durangensis Trel. (3)*. CHIH, DGO.

Nolina elegans Rose (4)*. ZAC.

Nolina erumpens (Torr.) S. Watson (3)*. COAH, CHIH, DGO.

Nolina humilis S. Watson (7)*. SLP.

Nolina juncea (Zucc.) MacBride (3,4). AGS, ZAC.

Nolina longifolia (Schult.) Hemsl. (8)*. OAX, PUE.

Nolina matapensis Wiggins (3)*. CHIH, SON.

Nolina microcarpa S. Watson (3). CHIH, DGO, SON.

Nolina nelsonii Rose $(4,7)$. TAMPS. 
Nolina palmeri S. Watson var. brandegeei Trel. (1)*. BC.

Nolina palmeri S. Watson var. palmeri $(0,1)$. BC.

Nolina parviflora (HBK) Hemsl. (3,4,7,8,9). D.F., HGO, JAL, MEX, MICH, NAY, OAX, PUE, QRO, SLP, VER, ZAC. Nolina parryi S. Watson $(0,1,3)$. BC, SON.

Nolina pumila Rose (3)*. NAY, SLP.

Nolina texana S. Watson var. compacta (Trel.) Johnston $(3,4)$. CHIH, DGO, SON.

Nolina watsonii (Bahr.) Hemsl. (4)*. SLP. 